\title{
Two-Photon Absorption by Impurity Scattering and Amplitude Mode in Conventional Superconductors
}

\author{
Takanobu Jujo * \\ Graduate School of Materials Science, Nara Institute of Science and Technology, \\ Ikoma, Nara 630-0101, Japan
}

(Received July 28, 2018)

We theoretically investigate the two-photon absorption spectrum of conventional s-wave superconductors. The calculation is carried out in the local limit at absolute zero. The direct excitation of quasiparticles is excluded under the condition that the frequency of the external field is lower than twice the magnitude of the superconducting gap. We calculate vertex corrections with the conserving approximation, and show that the amplitude mode is independent of the impurity scattering in the local limit. Because of this property, a diverging absorption edge appears in the dirty limit. We propose this behavior as a probe of the amplitude mode.

\section{Introduction}

The superconducting transition is accompanied by collective modes. ${ }^{1}$ Corresponding to degrees of freedom of the order parameter, there are phase and amplitude modes. The former couples with a density fluctuation and becomes a plasmon. ${ }^{2}$ This makes the experimental observation of the phase mode difficult. (The relative phase mode appears below the superconducting gap $(\Delta)$ in two-band superconductors ${ }^{3}$ or in the Josephson junction between two superconductors. ${ }^{4}$ This mode is used as a superconducting quantum bit. $\left.^{5}\right)$

The amplitude mode ${ }^{6,7}$ was investigated via phonon degrees of freedom in the superconducting charge-density-wave material ${ }^{8,9}$ because this mode does not directly interact with the external field (the interaction term is not given in the bilinear form). Therefore, the objects of this investigation have been restricted within specific materials. The development of the terahertz technique made the amplitude mode observable in other materials. This technique induces nonlinear responses, and an investigation by pump-probe spectroscopy has recently been performed. ${ }^{10}$

\footnotetext{
*E-mail address: jujo@ms.aist-nara.ac.jp
} 
By this method, an oscillation of the conductivity was observed by exciting quasiparticles by the pump pulse with its frequency comparable to $2 \Delta$. This type of observation of the transient state is a major method for studying the nonequilibrium superconductivity. In this paper, we show, however, that the amplitude mode is detectable in the two-photon absorption (TPA) spectrum without exciting extra degrees of freedom such as quasiparticles and phonons. Because a direct excitation of quasiparticles above the superconducting gap is excluded, this investigation can be performed in the steady state without destroying a superconducting state.

The conditions for our calculation are as follows. Absorption spectra are calculated in the dirty and local limits, ${ }^{11}$ which hold in a pump-probe experiment. ${ }^{10}$ Except for a calculation of the linear response, the frequency of the external field $(\omega)$ is set to be lower than $2 \Delta$, and then there is no direct excitation of quasiparticles across the superconducting gap. We also consider the case of the low-temperature limit $(T \rightarrow 0)$ in which the thermal excitation of quasiparticles is excluded. Under these conditions, the result of our calculation shows that the amplitude mode causes a divergent peak $(\sim 1 / \sqrt{\omega-\Delta})$ around the absorption edge $\omega=\Delta$, which is quantitatively estimated to be detectable.

In Sect. 2, the formulation to calculate the current density is given with a definition of the action. In Sect. 3, the one-particle Green function, the linear absorption function, and the TPA function without the vertex correction are calculated. In Sect. 4, the vertex correction is introduced and classified into the amplitude mode and impurity scattering. The TPA functions with these vertex corrections are calculated. In Sect. 5, numerical calculations are performed with use of the results in Sects. 3 and 4. In Sect. 6, a short summary is given. A brief discussion for a future study is also given. We set $\hbar=1$ in this paper.

\section{Formulation}

The response function is calculated with the use of the method of nonequilibrium Green's function. ${ }^{12,13}$ The definition of the current density is written as

$$
J_{q}(\omega)=\left.\frac{-i}{\sqrt{2}} \frac{\delta \ln Z[A]}{\delta A_{-q}^{q u}(-\omega)}\right|_{A^{q u} \rightarrow 0} .
$$

Here, $i=\sqrt{-1}$ and $Z[A]=\int \mathcal{D}[\psi, b] \mathrm{e}^{i S_{\psi, b}[A]}$. The action $S_{\psi, b}[A]=S_{\psi}+S_{b}+S_{\psi-A}+S_{\psi-b}+$ $S_{i m p}$ is given by the summation of terms for electrons $(\psi)\left[S_{\psi}=\int \mathrm{d} t \sum_{k, \sigma} \bar{\psi}_{k, \sigma}(t)\left(i \partial_{t}-\right.\right.$ $\left.\xi_{k}\right) \psi_{k, \sigma}(t)$ ( $k$ and $\sigma$ indicate the wave number and spin, respectively)], phonons $(b)\left[S_{b}=\right.$ 
$\left.\int \mathrm{d} t \sum_{q} \bar{b}_{q}(t)\left(i \partial_{t}-\omega_{q}\right) b_{q}(t)\right]$, the interaction between electrons and the external field $(A)$ $\left[S_{\psi-A}=\int \mathrm{d} t\left(N^{3}\right)^{-1 / 2} \sum_{k, q, \sigma} \bar{\psi}_{k+q, \sigma}(t) \mathrm{e} A_{q}(t) v_{k+q / 2} \psi_{k, \sigma}(t)\right.$ with $A_{q}(t)$ as the vector potential], the electron-phonon interaction $\left[S_{\psi-b}=\int \mathrm{d} t g_{p h}\left(N^{3}\right)^{-1 / 2} \sum_{k, q, \sigma} \bar{\psi}_{k+q, \sigma}(t)\left(b_{q}(t)+\right.\right.$ $\left.\bar{b}_{-q}(t)\right) \psi_{k, \sigma}(t)\left(g_{p h}\right.$ is the coupling constant)], and the impurity scattering for electrons $\left[S_{i m p}=\int \mathrm{d} t\left(N^{3}\right)^{-1 / 2} \sum_{k, k^{\prime}, \sigma} \bar{\psi}_{k, \sigma}(t) u_{k-k^{\prime}} \psi_{k^{\prime}, \sigma}(t)\right] .\left(N^{3}\right.$ is the number of sites. $\omega_{q}$ is the frequency of phonons. $\xi_{k}=k^{2} / 2 m-E_{F}$ is the kinetic energy of electrons measured from the Fermi energy $E_{F}$, and $v_{k}=\partial \xi_{k} / \partial k$. The direction of the external field is fixed and its index is omitted. $u_{k-k^{\prime}}$ represents the impurity potential and will be assumed to be isotropic.)

The integration $\left(\int \mathrm{d} t\right)$ is carried out on the forward and backward paths in time. ${ }^{13}$ The Coulomb interaction is omitted because it is not effective except for pushing up the phase mode to the plasmon. ${ }^{2} A^{q u}=\left(A^{+}-A^{-}\right) / \sqrt{2}$, in which $A^{+}$and $A^{-}$represent the vector potentials in the forward and backward directions in time, respectively. The physical external field is given by $A^{c l} / \sqrt{2}$ with $A^{c l}=\left(A^{+}+A^{-}\right) / \sqrt{2}$. (The definition of $A^{c l, q u}$ is different from that in Ref. 13 by a factor $\sqrt{2}$.)

\section{Response Function}

\subsection{Linear response}

First, we show the calculation of the linear response, and in this case, the current density is written as $J_{\omega}^{(1)}=-K_{\omega}^{(1)} A_{\omega}$ with $K_{\omega}^{(1)}=\mathrm{e}^{2}\left(4 \pi i N^{3}\right)^{-1} \sum_{k} \int \mathrm{d} \epsilon \operatorname{Tr}\left[\check{G}_{k}(\epsilon+\right.$ $\left.\omega) \check{G}_{k}(\epsilon) \check{1}^{q u}\right]\left(v_{k}\right)^{2}$. Here, $\check{1}^{q u}=\left(\begin{array}{cc}\hat{0} & \hat{1} \\ \hat{1} & \hat{o}\end{array}\right)(\hat{1}$ and $\hat{0}$ are the unit and null $2 \times 2$ matrix, respectively). In the calculation of the response function, we omit the diamagnetic term, which is proportional to $1 / m=\partial v_{k} / \partial k$ (the inverse of the electron mass) because it is negligible. ${ }^{14}$ (Hereafter, the index of the wave vector $q$ is omitted because we consider the local limit $q=0$.) The electronic Green's function in the Keldysh-Nambu space is

$$
\check{G}_{k}(\epsilon)=\left(\begin{array}{cc}
\hat{G}_{k}^{+}(\epsilon) & \hat{G}_{k}^{K}(\epsilon) \\
\hat{0} & \hat{G}_{k}^{-}(\epsilon)
\end{array}\right) .
$$

This is calculated with the Born approximation for the impurity scattering ${ }^{15}$ and the weak coupling approximation for the electron-phonon interaction, and the results are

$$
\hat{G}_{k}^{ \pm}(\epsilon)=\frac{\eta_{\epsilon}^{ \pm} \epsilon \hat{1}+\xi_{k} \hat{\tau}_{3}+\eta_{\epsilon}^{ \pm} \Delta \hat{\tau}_{1}}{\left(\eta_{\epsilon}^{ \pm} \epsilon\right)^{2}-\xi_{k}^{2}-\left(\eta_{\epsilon}^{ \pm} \Delta\right)^{2}}
$$

and $\hat{G}_{k}^{K}(\epsilon)=\tanh (\epsilon / 2 T)\left[\hat{G}_{k}^{+}(\epsilon)-\hat{G}_{k}^{-}(\epsilon)\right]$. Here, $\hat{\tau}_{1}=\left(\begin{array}{cc}0 & 1 \\ 1 & 0\end{array}\right), \hat{\tau}_{2}=\left(\begin{array}{cc}0 & -i \\ i & 0\end{array}\right)$, and $\hat{\tau}_{3}=\left(\begin{array}{cc}1 & 0 \\ 0 & -1\end{array}\right)$ are Pauli matrices, and $\eta_{\epsilon}^{ \pm}=1+i \alpha / X_{ \pm \epsilon}$ with $X_{\epsilon}=\operatorname{sgn}(\epsilon) \sqrt{\epsilon^{2}-\Delta^{2}} \theta(|\epsilon|-\Delta)+$ 
$i \sqrt{\Delta^{2}-\epsilon^{2}} \theta(\Delta-|\epsilon|)$ and $\alpha=n_{i} u^{2} m k_{F} / 2 \pi\left\{n_{i}\right.$ and $u$ are the concentration of impurities and the magnitude of the impurity potential, respectively. $k_{F}$ is the Fermi wave number. $\operatorname{sgn}(\epsilon)=\epsilon /|\epsilon|$, and $\theta(x)=\lim _{T \rightarrow 0}[1+\tanh (x / 2 T)] / 2$ is a step function $\}$. We consider the Einstein phonon with its frequency $\omega_{q}=\omega_{E}$, and in the weak coupling approximation $\left(\Delta \ll \omega_{E}\right),{ }^{16}$ the gap equation is written as

$$
\Delta \hat{\tau}_{1}=\frac{g_{p h}^{2}}{\omega_{E}} \frac{1}{N^{3}} \sum_{k} \int \frac{\mathrm{d} \epsilon}{2 \pi i} \hat{\tau}_{3} \hat{G}_{k}^{K}(\epsilon) \hat{\tau}_{3}
$$

The calculated result for the linear absorption spectrum (the real part of the conductivity; $\left.\operatorname{Re} \sigma_{\omega}^{(1)}=-\operatorname{Im} K_{\omega}^{(1)} / \omega\right)$ is written as

$$
\operatorname{Re} \sigma_{\omega}^{(1)}=\sigma_{\Delta} \frac{\alpha \Delta}{3 \pi^{2} \omega} \int_{\Delta-\omega / 2}^{-\Delta+\omega / 2} \mathrm{~d} \epsilon \sum_{s= \pm 1} \frac{s+\left(\epsilon^{2}-\omega^{2} / 4+\Delta^{2}\right) /\left(X_{\epsilon+\omega / 2} X_{\epsilon-\omega / 2}\right)}{\left(X_{\epsilon+\omega / 2}-s X_{\epsilon-\omega / 2}\right)^{2}+4 \alpha^{2}} \theta(\omega-2 \Delta),
$$

which is the same as that of previous works. ${ }^{17,18}$ Here, $\sigma_{\Delta}=\mathrm{e}^{2} k_{F}^{3} / m \Delta$, and the relaxation time $\tau$ in Ref. 18 corresponds to $1 / 2 \alpha$ in our calculation, and its value is roughly $\alpha \simeq 50 \sim 70$ in Ref. 10. In the local limit, the absorption occurs owing to the finite values of $\alpha$ and increases with $\alpha$ for small values of $\alpha(\alpha \lesssim \Delta)$. For large values of $\alpha$ $(\alpha \gg \Delta)$

$$
\operatorname{Re} \sigma_{\omega}^{(1)} \simeq \sigma_{0} \int_{\Delta-\omega / 2}^{-\Delta+\omega / 2} \mathrm{~d} \epsilon \frac{\epsilon^{2}-\omega^{2} / 4+\Delta^{2}}{\omega X_{\epsilon+\omega / 2} X_{\epsilon-\omega / 2}} \theta(\omega-2 \Delta) .
$$

Here, $\sigma_{0}=\mathrm{e}^{2} n_{e} \tau / m$ with $n_{e}=k_{F}^{3} / 3 \pi^{2}$ and $\tau=1 / 2 \alpha$. Then, the absorption decreases with increasing $\alpha$ and $\operatorname{Re} \sigma^{(1)} \propto 1 / \alpha$. It is shown below that there is a difference in the absorption edge between the linear and nonlinear responses. The absorption goes to zero for $\omega \rightarrow 2 \Delta$ (a vanishing absorption edge), as known from the above expression for $\operatorname{Re} \sigma_{\omega}^{(1)}$. This originates from a property of the vertex $v_{k} \hat{1}$. The vertex correction is not effective in the linear response because of the inversion symmetry, as discussed in Sect. 4.1.

\subsection{Third-order response without the vertex correction}

The third-order nonlinear response function is introduced to calculate the TPA spectrum. The current density in this case is given by $J_{\omega}^{(3)}=-K_{\omega}^{(3)} A_{\omega}$ with $K_{\omega}^{(3)}=K_{\omega}^{(3 n v)}+$ $K_{\omega}^{(3 v c)}$. ( $K_{\omega}^{(3 n v)}$ and $K_{\omega}^{(3 v c)}$ are defined below.) The third-order response function with no vertex correction is $K_{\omega}^{(3 n v)}=2\left(\tilde{K}_{\omega, \omega,-\omega}+\tilde{K}_{\omega,-\omega, \omega}+\tilde{K}_{-\omega, \omega, \omega}\right)|E|^{2} / \omega^{2}$ with $\tilde{K}_{\omega_{1}, \omega_{2}, \omega_{3}}=$ $\mathrm{e}^{4}\left(4 \pi i N^{3}\right)^{-1} \sum_{k} \int \mathrm{d} \epsilon \operatorname{Tr}\left[\check{G}_{k}\left(\epsilon+\omega_{1}+\omega_{2}+\omega_{3}\right) \check{G}_{k}\left(\epsilon+\omega_{2}+\omega_{3}\right) \check{G}_{k}\left(\epsilon+\omega_{3}\right) \check{G}_{k}(\epsilon) \check{1}^{q u}\right]\left(v_{k}\right)^{4}$. $(|E|$ is the magnitude of the electric field.) The absorption spectrum obtained by this 
process is given by

$\operatorname{Re} \sigma_{\omega}^{(3 n v)}=\sigma_{\Delta}\left(\frac{\mathrm{e} \xi_{0}|E|}{\omega}\right)^{2} \frac{\Delta^{3}}{5 \pi^{2} \omega} \int_{\Delta-\omega}^{-\Delta+\omega} \mathrm{d} \epsilon \sum_{s= \pm 1} s \operatorname{Re}\left(\frac{F_{A}^{s}+F_{A B}^{s}+F_{B}^{s}+2 F_{C}^{s}}{z_{0}\left(z_{0}+z_{1}\right)^{2}\left(z_{0}+z_{2}\right)^{2}\left(z_{1}+z_{2}\right)}\right) \theta_{\omega}^{<}$.

Here, $\theta_{\omega}^{<}=\theta(\omega-\Delta) \theta(2 \Delta-\omega)$ (the first and second $\theta^{\prime}$ s correspond to the absence of thermal excitation and a restriction for our calculation, respectively), $\xi_{0}=v_{F} / \Delta$ $\left(v_{F}=k_{F} / m\right.$ is the Fermi velocity), $z_{j}=\zeta_{j}+\alpha(j=0,1,2), \zeta_{0}=-i X_{\epsilon}, \zeta_{1}=i X_{\epsilon+\omega}$, $\zeta_{2}=-s i X_{\epsilon-\omega}, F_{A}^{s}=\left[2 z_{0}\left(z_{0}+z_{1}+z_{2}\right)^{2}+z_{1} z_{2}\left(z_{1}+z_{2}\right)\right]\left[\epsilon^{4}+6 \epsilon^{2} \Delta^{2}+\Delta^{4}-\omega^{2}\left(\epsilon^{2}+\Delta^{2}\right)\right] / \zeta_{1} \zeta_{2} \zeta_{0}^{2}$, $F_{A B}^{s}=\left[2 z_{0} z_{1} z_{2}+z_{1} z_{2}\left(z_{1}+z_{2}\right)\right]\left(\epsilon^{2}-\omega^{2}-\Delta^{2}\right) / \zeta_{1} \zeta_{2}, F_{B}^{s}=-2 z_{0}\left(z_{0}^{2}-z_{1} z_{2}\right)$, and $F_{C}^{s}=$ $2\left[z_{0} z_{1}\left(2 z_{0}+z_{1}+z_{2}\right)\right]\left[\epsilon(\epsilon+\omega)+\Delta^{2}\right] / \zeta_{1} \zeta_{0}$. These terms correspond to various matrix elements, and the term with $F_{A B}^{s}$ causes a finite absorption at the band edge $\omega=\Delta$. (The other terms have vanishing absorption edges.) This behavior is different from that of the linear response in which the absorption vanishes at the band edge $(\omega=$ $2 \Delta$ ) owing to the symmetry $v_{k}=-v_{-k}$. The nonlinear process changes this behavior because of the existence of intermediate states. The dependence of the absorption on $\alpha$ is significant compared with that of the linear response, and $\operatorname{Re} \sigma^{(3 n v)} \propto 1 / \alpha^{3}$ for $\alpha \gg \Delta$. Therefore, $\operatorname{Re} \sigma^{(3 n v)}$ is negligible in the dirty limit as compared with terms including vertex corrections calculated below.

\section{Vertex Correction}

The current density with the vertex correction is also derived from Eq. (1). The diagram of the response function is shown in Fig. 1(a). The vertex correction is introduced, consistent with the conserving approximation. ${ }^{19}$ The irreducible four-point vertex is obtained from the self-energy. The latter is already included in the above calculations and its diagram is shown in Fig. 1(b). From this interaction term, the vertex correction is obtained by solving an integral equation with its diagrammatic representation given in Fig. 1(c). We divide this into two terms as in Fig. 2(a); one contains the electron-phonon interaction [Figs. 2(b) and 2(d)] and the other does not contain it as a four-point vertex [Fig. 2(c)]. The former is called a vertex correction by the amplitude mode because it leads to the variation of the superconducting gap as shown below, and the response function is written as $K_{\omega}^{(3 a m)}$, which contains $\Pi$ of Fig. 2(b) as the vertex correction. The latter is the vertex correction by the impurity scattering because it contains only the impurity scattering as a four-point vertex, and the response function is written as $K_{\omega}^{(3 i m)}$, which contains $\Lambda$ of Fig. $2(\mathrm{c})$ as the vertex correction. Then, the 


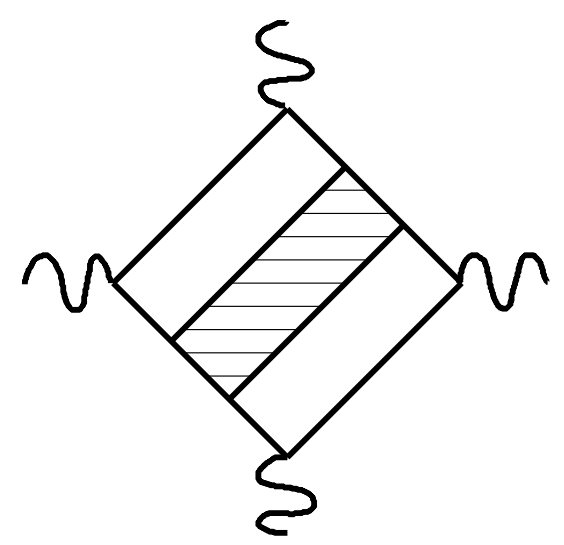

(a)
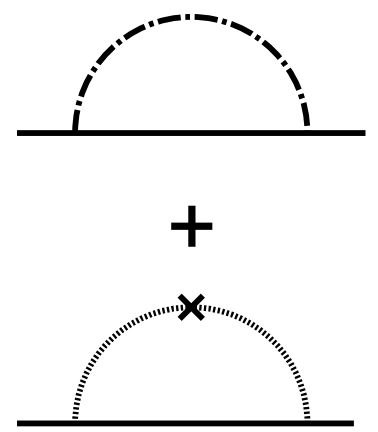

(b)

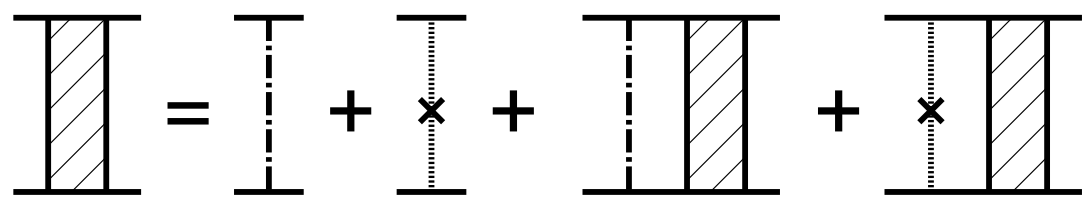

(c)

Fig. 1. (a) Diagram of a third-order nonlinear response function with vertex corrections. The wavy lines, solid lines, and shaded square represent the external field, electrons, and vertex correction, respectively. (b) Diagram of the self-energy. The dashed line represents the electron-phonon interaction. The dotted line with a cross indicates the impurity scattering. (c) Diagrammatic representation of the integral equation for the vertex correction.

response function with the vertex correction is written as $K_{\omega}^{(3 v c)}=K_{\omega}^{(3 a m)}+K_{\omega}^{(3 i m)}$.

\subsection{Vertex correction by the amplitude mode}

First, we show the calculation of $K_{\omega}^{(3 \mathrm{am})}$. The weak coupling approximation is introduced for the electron-phonon interaction as in the case of the self-energy. (This approximation corresponds to neglecting the inelastic scattering, which is considered to be small at low temperatures.) Then, the result is written as

$$
K_{\omega}^{(3 a m)}=\frac{\mathrm{e}^{4}|E|^{2}}{2 \omega^{2}} \frac{g_{p h}^{2} t}{\omega_{E}} \boldsymbol{\chi}_{\omega}^{(S)} \check{\mathcal{T}}_{\bar{M}_{\omega}} \boldsymbol{\chi}_{\omega}^{(K)} \theta_{\omega}^{<} .
$$

Here, $\quad \check{\mathcal{T}}=\left(\begin{array}{cc}\hat{1} & 0 \\ \hat{0} & -\hat{\tau}_{1}\end{array}\right), \quad \boldsymbol{\chi}_{\omega}^{(K)}=\sum_{s= \pm} s\left(\boldsymbol{h}_{-\omega}^{++s}+\boldsymbol{h}_{0}^{+s-}+\boldsymbol{h}_{\omega}^{s--}\right)$, and $\boldsymbol{\chi}_{\omega}^{(S)}=$ $\sum_{s, u= \pm} s\left(\boldsymbol{h}_{-\omega}^{+u s}+\boldsymbol{h}_{0}^{u s u}+\boldsymbol{h}_{\omega}^{s u-}\right)$. The vector ${ }^{t} \boldsymbol{h}=\left(h_{11}, h_{22}, h_{12}, h_{21}\right)$ is made from the 
(a)
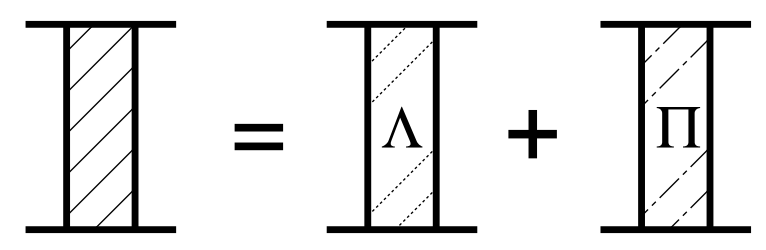

(b)

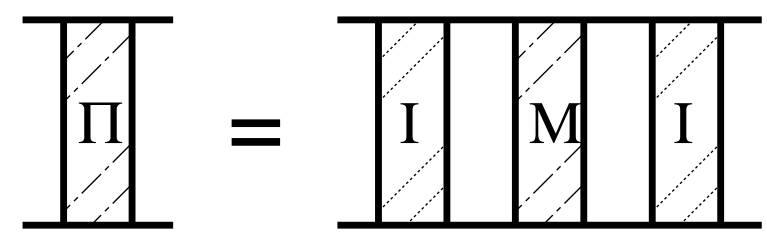

(c)
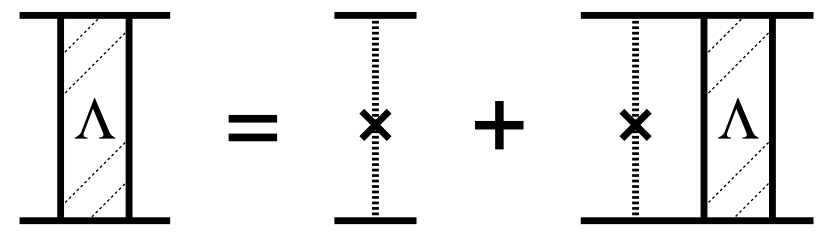

(d)
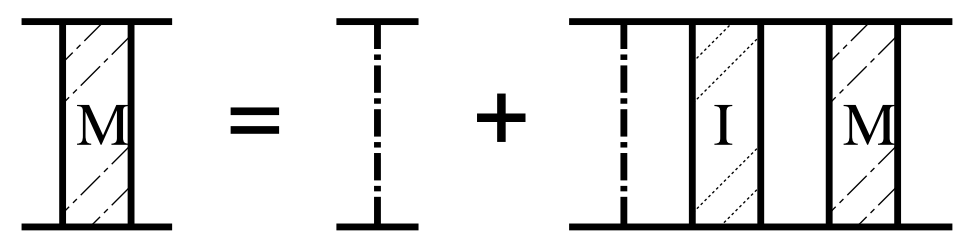

Fig. 2. (a) Division of the four-point vertex into two terms ( $\Lambda$ and $\Pi$ ). (b) Rewriting the term that contains the electron-phonon interaction as a four-point vertex. (c) Integral equation for the vertex correction by the impurity scattering. (d) Integral equation for the vertex correction by the electron-phonon interaction. The definition of $I$ is given in Sect. 4.1 as $\check{I}$ [Eq. (5)], and $\Lambda$ is equal to $\check{I}-\check{1}$. The expression for $\check{M}$ is also given in Sect. 4.1 [Eq. (6)].

corresponding components of a $2 \times 2$ matrix $\hat{h}$ ( ${ }^{t}$ indicates a transposition).

$$
\begin{array}{r}
\left(\hat{h}_{x}^{a b c}\right)_{i, j}=\frac{1}{N^{3}} \sum_{k} v_{k}^{2} \int \frac{\mathrm{d} \epsilon}{2 \pi i} \tanh \left(\frac{\epsilon+x}{2 T}\right) \sum_{n, l}\left(\check{I}_{\epsilon+\omega, \epsilon-\omega}^{a, c}\right)_{n, l}^{i, j} \\
\times\left[\hat{\tau}_{3} \hat{G}_{k}^{a}(\epsilon+\omega) \hat{G}_{k}^{b}(\epsilon) \hat{G}_{k}^{c}(\epsilon-\omega) \hat{\tau}_{3}\right]_{n, l}
\end{array}
$$

$(a, b, c=+$ or - and $i, j, n, l=1,2$ designate the elements of a matrix) with the vertex correction by the impurity scattering;

$$
\check{I}_{\epsilon+\omega, \epsilon-\omega}^{a, c}=\left(\check{1}-n_{i} u^{2} \check{m}_{\epsilon+\omega, \epsilon-\omega}^{a, c}\right)^{-1} .
$$


Here, $\check{1}$ is a $4 \times 4$ unit matrix and

$$
\left(\check{m}_{\epsilon+\omega, \epsilon-\omega}^{a, c}\right)_{n, l}^{i, j}=\frac{1}{N^{3}} \sum_{k}\left[\hat{\tau}_{3} \hat{G}_{k}^{a}(\epsilon+\omega)\right]_{i, n}\left[\hat{G}_{k}^{c}(\epsilon-\omega) \hat{\tau}_{3}\right]_{l, j} .
$$

The vertex correction by the electron-phonon interaction is

$$
\check{M}_{\omega}=\left(\check{1}-\check{R}_{-\omega}^{+,+}+\check{R}_{-\omega}^{+,-}-\check{R}_{\omega}^{+,-}+\check{R}_{\omega}^{-,-}\right)^{-1}
$$

with

$$
\check{R}_{x}^{a, b}=\frac{g_{p h}^{2}}{\omega_{E}} \int \frac{\mathrm{d} \epsilon}{2 \pi i} \tanh \left(\frac{\epsilon+x}{2 T}\right) \check{I}_{\epsilon+\omega, \epsilon-\omega}^{a, b} \check{m}_{\epsilon+\omega, \epsilon-\omega}^{a, b} .
$$

Equation (4) is rewritten as $K_{\omega}^{(3 a m)}=\left(\mathrm{e}^{2} / 2\right)^{t} \boldsymbol{\chi}_{\omega}^{(S)} \check{\mathcal{T}} \boldsymbol{\delta} \boldsymbol{\Delta}_{\omega} \theta_{\omega}^{<}$. Here, $\boldsymbol{\delta} \boldsymbol{\Delta}_{\omega}=$ $\left(g_{p h}^{2} / \omega_{E}\right)(\mathrm{e}|E| / \omega)^{2} \check{M}_{\omega} \chi_{\omega}^{(K)}$ is interpreted as a nonequilibrium part of the self-energy because it includes the effect of the external field. $\hat{\chi}_{\omega}^{(K, S)}$ and $\delta \hat{\Delta}_{\omega}$ are proportional to $\hat{\tau}_{1}$ because of the particle-hole symmetry, and $\hat{\tau}_{1}$ represents an off-diagonal order as is known from expressions of the Green's function of electrons and the gap equation. Therefore, $\boldsymbol{\delta} \boldsymbol{\Delta}_{\omega}$ represents the variation of the superconducting gap under the external field, and the equation for $\boldsymbol{\delta} \boldsymbol{\Delta}_{\omega}$ is rewritten as $\boldsymbol{\delta} \boldsymbol{\Delta}_{\omega}=(\mathrm{e}|E| / \omega)^{2} \boldsymbol{\chi}_{\omega}^{(K)} / \Gamma_{q}^{(a m)}(2 \omega)$. Here, $1 / \Gamma_{q}^{(a m)}(2 \omega)=\left[\left(\check{M}_{\omega}\right)_{1,2}^{1,2}+\left(\check{M}_{\omega}\right)_{2,1}^{1,2}\right] g_{p h}^{2} / \omega_{E}$, and $\Gamma_{q}^{(a m)}(2 \omega)$ represents an amplitude mode. ${ }^{9}$ This is calculated as

$$
\begin{aligned}
\Gamma_{q}^{(a m)}(2 \omega)= & \frac{m k_{F}}{2 \pi^{2}} \int \mathrm{d} \epsilon \tanh \left(\frac{\epsilon}{2 T}\right)\left[\frac{1}{X_{\epsilon}^{(r)}}\right. \\
& \left.-\sum_{s= \pm 1} \frac{f_{s}(q)}{1-2 i \alpha f_{s}(q)}\left(\frac{\epsilon(\epsilon+2 \omega)+\Delta^{2}}{X_{\epsilon+2 \omega} X_{\epsilon}^{(r)}}+s\right)\right] .
\end{aligned}
$$

Here, $f_{s}(q)=2^{-1} \int_{-1}^{1} \mathrm{~d}(\cos \theta) /\left(X_{\epsilon+2 \omega}+s X_{\epsilon}^{(r)}+2 i \alpha-v_{F} q \cos \theta\right)$ and $X_{\epsilon}^{(r)}=\operatorname{Re} X_{\epsilon}$.

In TPA, there exists another type of coupling vertex with external fields: $\hat{\tau}_{3} \partial v_{k} / \partial k$ (the diamagnetic term). In this case, the quantity corresponding to $\hat{\chi}_{\omega}^{(K)}$ is proportional to linear superpositions of $\hat{\tau}_{3}$ and $\hat{\tau}_{2}$. This represents density $\left(\hat{\tau}_{3}\right)$ and phase $\left(\hat{\tau}_{2}\right)$ fluctuations, and these result in a plasma oscillation. This term is negligible because of its high-energy excitation energy and the smallness of this type of vertex. ${ }^{14}$ In the case of the linear response, the quantity corresponding to $\hat{\chi}_{\omega}^{(K)}$ includes the single vertex $v_{k}$ in contrast to $v_{k}^{2}$ in TPA. Therefore, the amplitude mode does not contribute to the linear absorption because of the inversion symmetry $v_{-k}=-v_{k}$. This holds true even if the nonlocality $(q \neq 0)$ is introduced..$^{20}$ 
The resulting absorption spectrum is given by

$$
\operatorname{Re} \sigma_{\omega}^{(3 a m)}=\sigma_{\Delta}\left(\frac{\mathrm{e} \xi_{0}|E|}{\omega}\right)^{2} \frac{2 \Delta}{\omega} \operatorname{Im}\left[\frac{\chi_{\omega}^{\prime 2}}{\Gamma_{q=0}^{\prime}(2 \omega)}\right] \theta_{\omega}^{<} .
$$

Here, $\Gamma_{q}^{\prime}(2 \omega)=\left(m k_{F}\right)^{-1} \Gamma_{q}^{(a m)}(2 \omega)$ and $\chi_{\omega}^{\prime}=m \Delta k_{F}^{-3}\left(\hat{\chi}_{\omega}^{(K)}\right)_{1,2}$ are dimensionless quantities. $\left(\hat{\chi}_{\omega}^{(K)}=\hat{\chi}_{\omega}^{(S)} / 2\right.$ at $T=0$.) From Eq. (7), $\operatorname{Re} \Gamma_{q=0}^{\prime}(2 \omega)=$ $\left(1 / \pi^{2}\right) \sqrt{1-\Delta^{2} / \omega^{2}} \operatorname{arcsinh} \sqrt{(\omega / \Delta)^{2}-1}$ and $\operatorname{Im} \Gamma_{q=0}^{\prime}(2 \omega)=-(1 / 2 \pi) \sqrt{1-\Delta^{2} / \omega^{2}}$ for $\omega \geq \Delta$. This expression of $\Gamma_{q=0}^{\prime}(2 \omega)$ shows that, in the local limit, the amplitude mode is independent of the impurity scattering when its vertex correction is included, consistent with a conservation law. This property is similar to the fact that nonmagnetic impurities are ineffective on the gap in conventional $s$-wave superconductors. ${ }^{21}$ This result is consistent with an observation of the long-time $(\gg \tau)$ oscillation of the amplitude mode, ${ }^{10}$ and gives an explanation for this behavior.

Although the amplitude mode is independent of $\alpha$, the absorption by this mode depends on the impurity scattering. In Eq. (8), $\chi^{\prime}$ is roughly proportional to $1 / \alpha$ for $\alpha \gg \Delta$

$$
\operatorname{Re} \chi_{\omega}^{\prime} \simeq \frac{-\Delta^{2}}{6 \pi^{2} \alpha} \int_{\omega-\Delta}^{\infty} \mathrm{d} \epsilon \operatorname{Im}\left(\frac{3 \epsilon^{2}-\omega^{2}+\Delta^{2}+X_{\epsilon+\omega} X_{\epsilon-\omega}}{X_{\epsilon} X_{\epsilon+\omega} X_{\epsilon-\omega}\left(X_{\epsilon+\omega}+X_{\epsilon-\omega}\right)}\right)
$$

and $\operatorname{Im} \chi_{\omega}^{\prime} \simeq-(\Delta / 12 \pi \alpha) \sqrt{1-\Delta^{2} / \omega^{2}}$. Then, $\operatorname{Re} \sigma^{(3 a m)} \propto 1 / \alpha^{2}$. The effect of the amplitude mode on the absorption spectrum is clearly observed at the band edge, which is $\operatorname{Re} \sigma^{(3 a m)} \propto 1 / \sqrt{\omega-\Delta}$ around $\omega \simeq \Delta$ because $\operatorname{Re} \Gamma_{q=0}^{\prime}(2 \omega) \propto \omega-\Delta$, $\operatorname{Im} \Gamma_{q=0}^{\prime}(2 \omega) \propto \sqrt{\omega-\Delta}$, and $\chi_{\omega}^{\prime} \simeq \operatorname{Re} \chi_{\Delta}^{\prime} \neq 0$ for $\omega \rightarrow \Delta$. The amplitude mode appears not around $\omega=2 \Delta$ but around $\omega=\Delta$. This is because the TPA results from two photons with the frequency of a photon being $\omega$, and $\omega=\Delta$ means $2 \omega=2 \Delta$. Here, "two photons" does not indicate the quantized electromagnetic field, but the second order of the external field (the excitation by $A_{\omega}^{2}$ ).

\subsection{Vertex correction by the impurity scattering}

$K_{\omega}^{(3 i m)}$ is derived in a similar way. In the case of $\omega<2 \Delta$ and $T \rightarrow 0$, the finite contribution to $\operatorname{Im} K^{(3)}$ comes from the term that includes a factor such as $\tanh \left(\epsilon_{-} / 2 T\right)-\tanh \left(\epsilon_{+} / 2 T\right)\left(\epsilon_{ \pm}=\epsilon \pm \omega\right)$. Then, the calculated result for the absorption spectrum is written as

$$
\operatorname{Re} \sigma_{\omega}^{(3 i m)}=\sigma_{\Delta}\left(\frac{\mathrm{e} \xi_{0}|E|}{\omega}\right)^{2} \frac{\alpha \Delta^{3}\left(2 F_{\omega}^{\prime}+F_{\omega}^{\prime \prime}\right)}{9 \pi^{2} \omega} \theta_{\omega}^{<} .
$$


Here, $\quad F_{\omega}^{\prime} \quad=\quad \int_{\Delta-\omega}^{-\Delta+\omega} \mathrm{d} \epsilon^{t} \boldsymbol{\kappa}_{\epsilon, \epsilon+\omega, \epsilon}^{+i+} \check{\mathcal{T}} \check{I}_{\epsilon, \epsilon}^{+,+} \boldsymbol{\kappa}_{\epsilon, \epsilon-\omega, \epsilon}^{+i+} \quad$ and $\quad F_{\omega}^{\prime \prime}=$

$-\int_{\Delta-\omega}^{-\Delta+\omega} \mathrm{d} \epsilon \sum_{s= \pm} s \operatorname{Re}\left({ }^{t} \boldsymbol{\kappa}_{\epsilon_{-}, \epsilon, \epsilon_{+}}^{s r+} \check{\mathcal{T}}_{\epsilon_{\epsilon_{+}, \epsilon_{-}}^{+, s}} \boldsymbol{\kappa}_{\epsilon_{+}, \epsilon, \epsilon_{-}}^{+r s}\right) \quad$ with $\quad \hat{\kappa}_{\epsilon_{1}, \epsilon_{2}, \epsilon_{3}}^{s_{1} c s_{2}}=$ $\int \mathrm{d} \xi_{k} \hat{\tau}_{3} \hat{G}_{k}^{s_{1}}\left(\epsilon_{1}\right) \mathcal{C}\left[\hat{G}_{k}^{+}\left(\epsilon_{2}\right)\right] \hat{G}_{k}^{s_{2}}\left(\epsilon_{3}\right) \hat{\tau}_{3} / \pi(\mathcal{C}=\operatorname{Re}$, Im for $c=r, i$, respectively $)$. The analytic calculation shows that $\operatorname{Re} \sigma_{\omega}^{(3 i m)} \propto 1 / \alpha^{2}$ for $\alpha \gg \Delta$. The absorption edge at $\omega=\Delta$ is finite as in the case of $\operatorname{Re} \sigma_{\omega}^{(3 n v)}$.

\section{Numerical Calculations}

Numerical calculations are carried out for Eqs. (2), (3), (8), and (9) for general values of $\alpha$ without taking $\alpha \gg \Delta$. The results of numerical calculations for $\operatorname{Re} \sigma_{\omega}^{(3)} / \sigma_{(\alpha, E)}$ are shown in Fig. 3(a). Here, $\operatorname{Re} \sigma_{\omega}^{(3)}=\operatorname{Re} \sigma_{\omega}^{(3 n v)}+\operatorname{Re} \sigma_{\omega}^{(3 a m)}+\operatorname{Re} \sigma_{\omega}^{(3 i m)}$ and $\sigma_{(\alpha, E)}=$ $\sigma_{\Delta}\left(\mathrm{e} \xi_{0}|E| / \alpha\right)^{2}$. As $\operatorname{Re} \sigma_{\omega}^{(3)} \propto 1 / \alpha^{2}, \operatorname{Re} \sigma_{\omega}^{(3)} / \sigma_{(\alpha, E)}$ becomes independent of $\alpha$ for $\alpha \gg \Delta$. Although this behavior is indicated by an analytical calculation, numerical calculations show that $\operatorname{Re} \sigma_{\omega}^{(3 \mathrm{am})}$ becomes predominant over $\operatorname{Re} \sigma_{\omega}^{(3 \mathrm{im})}$ for large values of $\alpha$, as shown in Fig. 3(b). This originates from the $\omega$-dependence of $\chi_{\omega}^{\prime}$, which varies with $\alpha$.

The dependences of $1 / \Gamma_{q=0}^{\prime}(2 \omega)$ and $\chi_{\omega}^{\prime 2}$ [in Eq. (8)] on $\omega$ are shown in Figs. 4(a) and 4(b) to clarify the contribution of the amplitude mode to the TPA spectrum. These dependences of $\Gamma^{\prime}$ and $\chi^{\prime}$ on $\omega$ are the same as expected in Sect. 4.1. $\operatorname{Im}\left[1 / \Gamma_{q=0}^{\prime}(2 \omega)\right]$ indicates the spectral weight of the amplitude mode, and $\operatorname{Re} \sigma_{\omega}^{(3 a m)} \propto$ $\operatorname{Im}\left(\chi_{\omega}^{\prime}\right)^{2} \operatorname{Re}\left[1 / \Gamma_{q=0}^{\prime}(2 \omega)\right]+\operatorname{Re}\left(\chi_{\omega}^{\prime}\right)^{2} \operatorname{Im}\left[1 / \Gamma_{q=0}^{\prime}(2 \omega)\right]$ from Eq. (8). This shows that the predominant contribution to the TPA spectrum around $\omega \simeq \Delta$ results from the amplitude mode because $\operatorname{Re}\left(\chi_{\omega}^{\prime}\right)^{2}$ is larger than $\operatorname{Im}\left(\chi_{\omega}^{\prime}\right)^{2}$ for this region. For $\omega<\Delta$, $\operatorname{Im} \Gamma_{q=0}^{\prime}(2 \omega)=0$ and $\operatorname{Im} \chi_{\omega}^{\prime}=0$ because of the absence of thermal excitation in the low-temperature limit, and then the TPA vanishes in this region. In the local limit $(q=0)$, the dependence of $\Gamma_{q}^{\prime}(2 \omega)$ on $q$ (the dispersion relation of the amplitude mode) is irrelevant to the TPA spectrum.

The variation of $\operatorname{Re} \sigma_{\omega}^{(3)}$ from the clean $(\alpha / \Delta \ll 1)$ to dirty $(\alpha / \Delta>1)$ cases is shown in Fig. 5(a). $\left[\sigma_{(\Delta, E)}=\sigma_{\Delta}\left(\mathrm{e} \xi_{0}|E| / \Delta\right)^{2}\right.$ in the denominator is independent of $\alpha$.] There is no absorption in the clean limit $(\alpha / \Delta \rightarrow 0)$ as noted above, ${ }^{11}$ and the TPA increases with increasing $\alpha$ in the case of $\alpha / \Delta \ll 1$. In the dirty case, the TPA decreases with increasing $\alpha$ as noted in previous sections. The reason for this is that the absorption occurs in the wider $\omega$ range as $\alpha$ increases, which is also seen in the linear absorption in Fig. 5(b). This shift of the spectral weight contributes to a relative predominance of the amplitude mode in the TPA around $\omega \simeq \Delta$ for the dirty limit.

We quantitatively estimate $\operatorname{Re} \sigma_{\omega}^{(3)} / \sigma_{0}$, which is equal to 

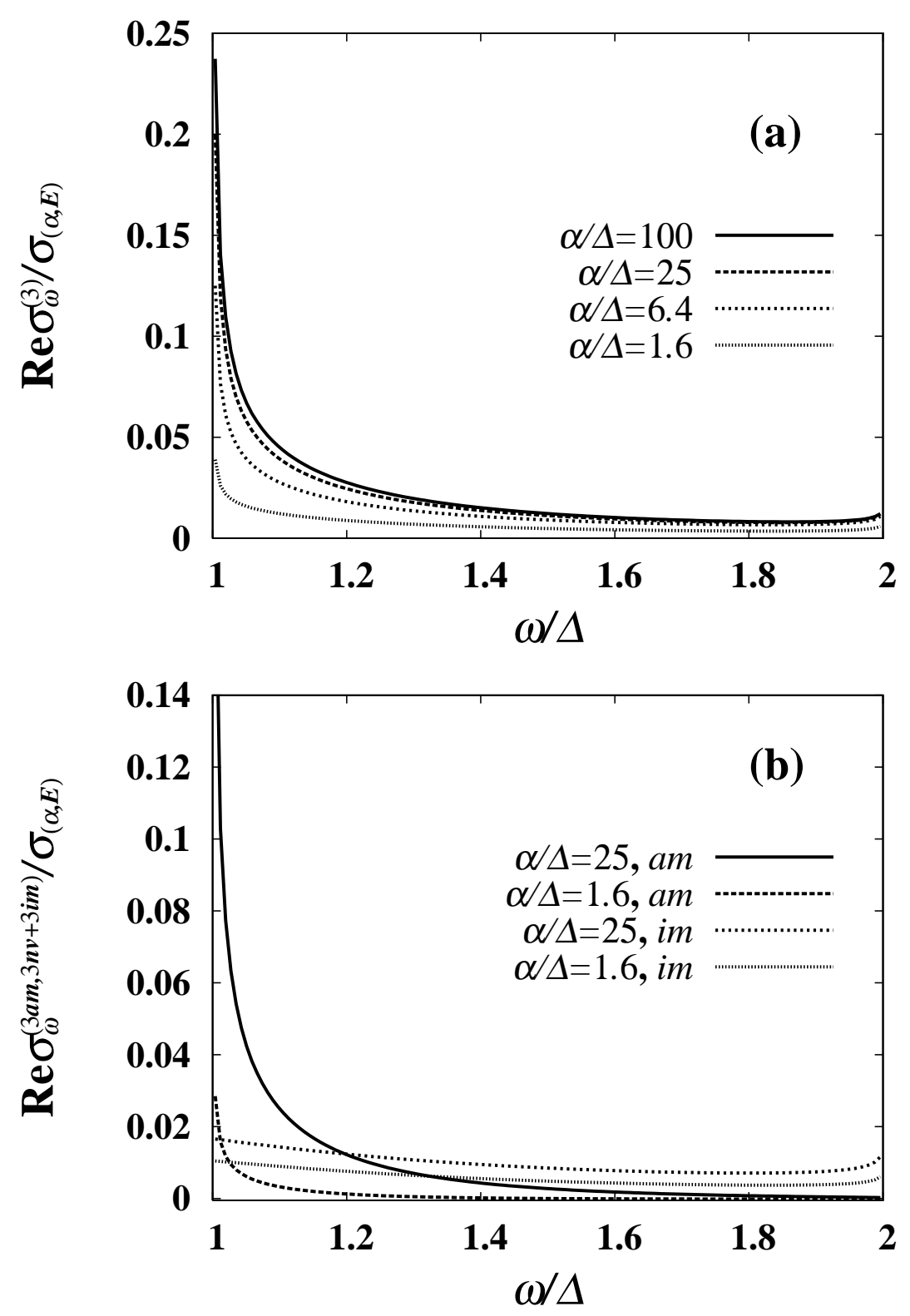

Fig. 3. (a) Dependence of $\operatorname{Re} \sigma_{\omega}^{(3)} / \sigma_{(\alpha, E)}$ on $\omega$ for several values of $\alpha$. (b) Dependences of $\operatorname{Re} \sigma_{\omega}^{(3 a m)} / \sigma_{(\alpha, E)}($ noted as $a m)$ and $\left(\operatorname{Re} \sigma_{\omega}^{(3 n v)}+\operatorname{Re} \sigma_{\omega}^{(3 i m)}\right) / \sigma_{(\alpha, E)}$ (noted as $i m$ ) on $\omega$ for $\alpha / \Delta=1.6$ and 25 .

$6 \pi^{2}\left[\left(\mathrm{e} \xi_{0}|E|\right)^{2} / \alpha \Delta\right]\left(\operatorname{Re} \sigma_{\omega}^{(3)} / \sigma_{(\alpha, E)}\right)$ and proportional to $|E|^{2} / \alpha$ for $\alpha \gg \Delta$. If we put $|E|=5 \mathrm{kV} / \mathrm{cm}, \xi_{0} / \pi=5 \mathrm{~nm}, \Delta=2.5 \mathrm{meV}$, and $\alpha / \Delta=100$, then $\left(\operatorname{Re} \sigma_{\omega}^{(3)} / \sigma_{0}\right) \simeq 5.8 \times\left(\operatorname{Re} \sigma_{\omega}^{(3)} / \sigma_{(\alpha, E)}\right)$. This indicates that the TPA by the amplitude mode can be observed in roughly the same magnitude as the linear response. Because this calculation is based on a perturbative expansion by the external field, the magnitude of the electric field should be adjusted so that the $\operatorname{Re} \sigma^{(3)} / \operatorname{Re} \sigma^{(1)}$ ratio is 

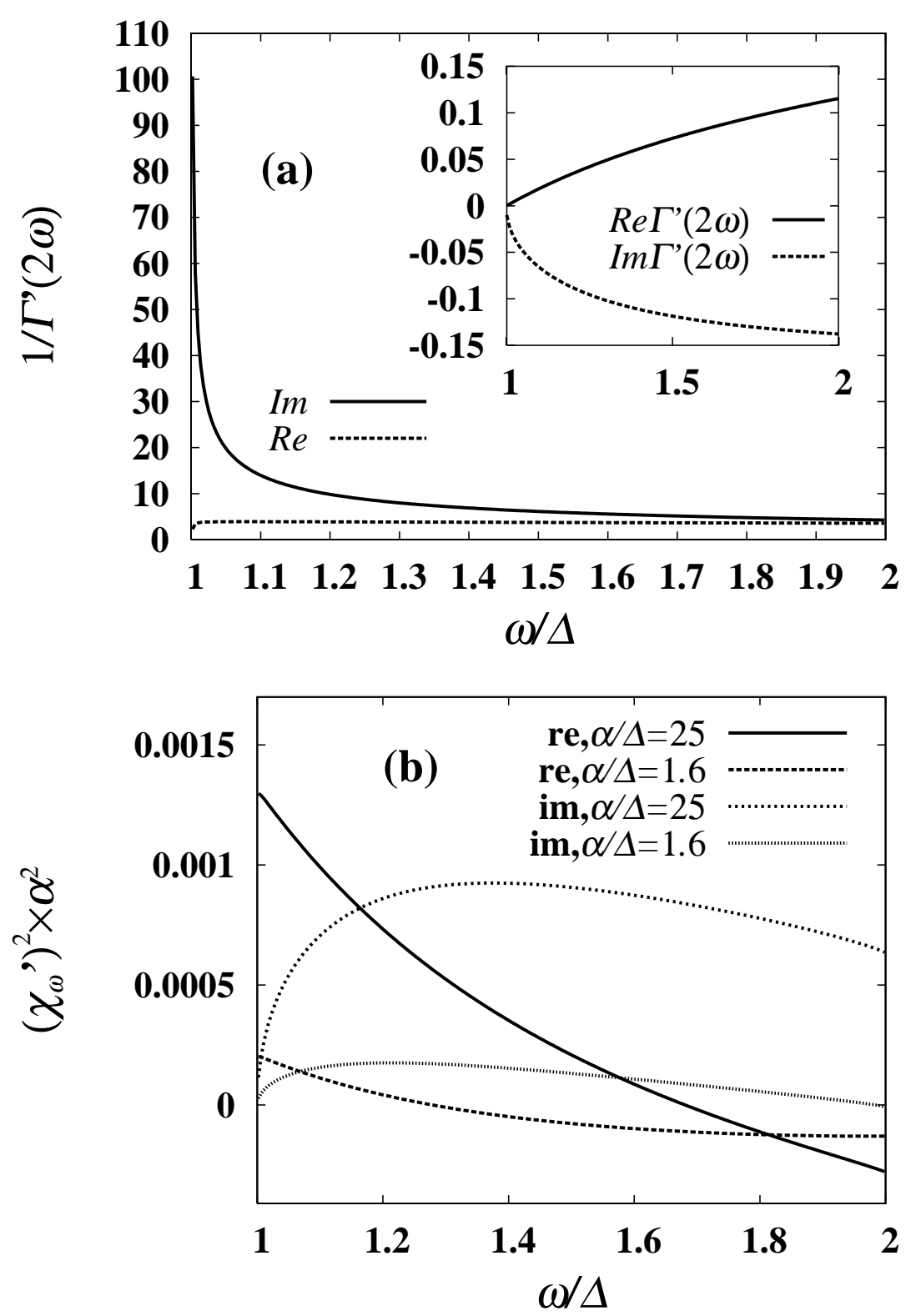

Fig. 4. (a) Dependences of the real (noted as Re) and imaginary $(\operatorname{Im})$ parts of $1 / \Gamma_{q=0}^{\prime}(2 \omega)$ on $\omega$. The inset shows $\omega$-dependences of $\operatorname{Re}\left[\Gamma_{q=0}^{\prime}(2 \omega)\right]$ and $\operatorname{Im}\left[\Gamma_{q=0}^{\prime}(2 \omega)\right]$. (b) Dependences of the real (noted as re) and imaginary (im) parts of $\alpha^{2} \chi_{\omega}^{\prime 2}$ on $\omega$ for $\alpha / \Delta=1.6$ and 25.

smaller than 1 for the validity of this theory.

\section{Summary and Discussion}

We calculated the TPA spectrum in dirty superconductors. As for the absorption edge and the dependence on the impurity scattering, there are differences between the linear and nonlinear responses. In the dirty limit, the term with vertex corrections gives a predominant contribution to the TPA. Although the finite absorption generally 

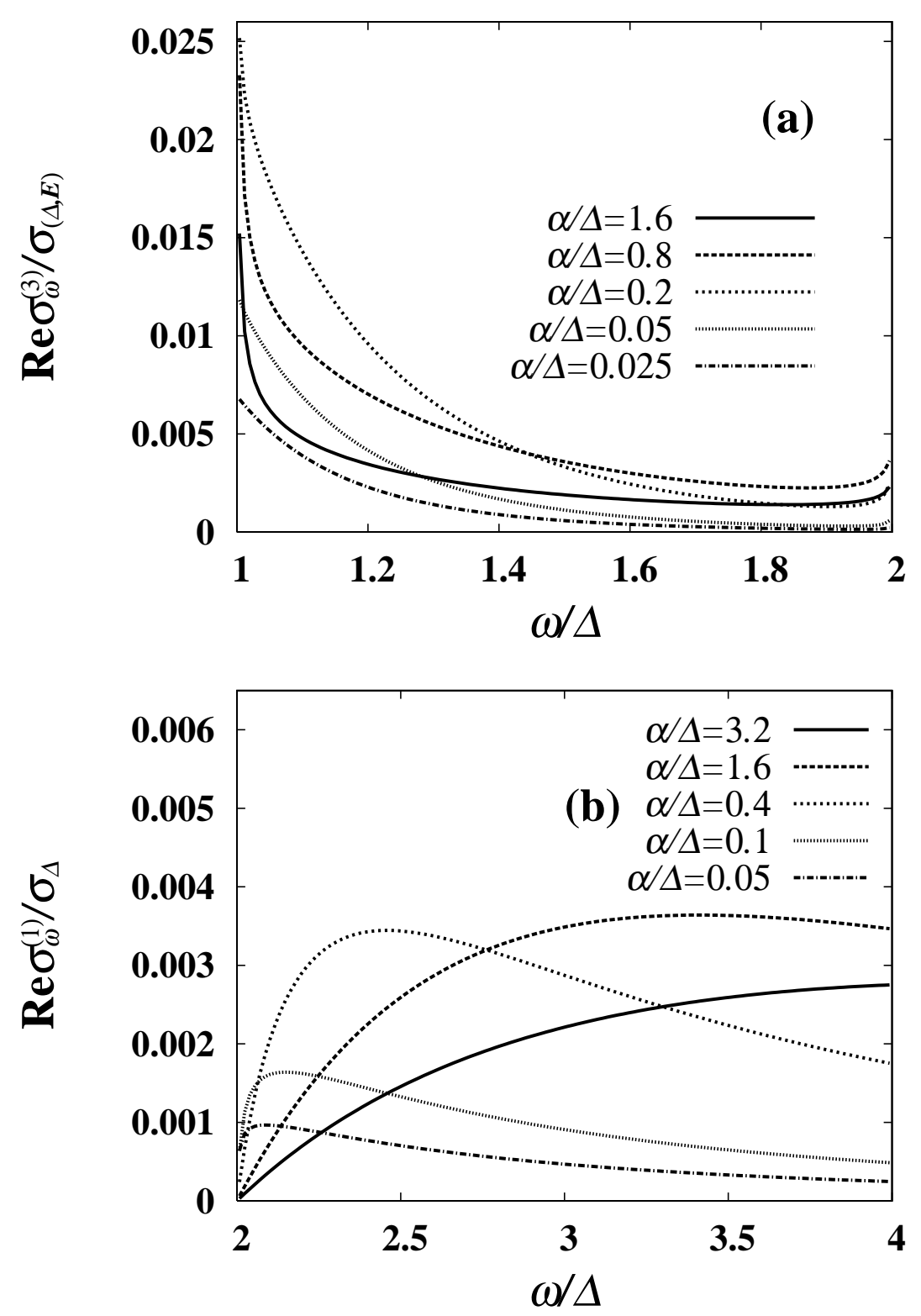

Fig. 5. Dependences of (a) $\operatorname{Re} \sigma_{\omega}^{(3)} / \sigma_{(\Delta, E)}$ and (b) $\operatorname{Re} \sigma_{\omega}^{(1)} / \sigma_{\Delta}$ on $\omega$ for several values of $\alpha$.

exists at the band edge in the TPA spectrum, the amplitude mode is clearly observed because of a singular behavior as $1 / \sqrt{\omega-\Delta}$. This mode is not suppressed in the dirty limit because a self-energy effect by the impurity scattering is canceled out with its vertex correction. Our result shows that the TPA would provide a probe to observe the amplitude mode experimentally.

Although we consider the steady state, our method can be extended to investigate the transient state by introducing the integration over frequencies of the external field. In the latter case, the restriction on $\omega(\omega<2 \Delta)$ is removed because of the finite 
radiation time. This type of study would give a direct comparison of a theory to the pump-probe spectroscopy. ${ }^{10}$

Investigating various types of interaction is also a possible application of our method. The method of calculating the vertex correction with the conserving approximation can be extended beyond the mean field approximation (for example, strong coupling effect). This is in contrast to a method using the Hubbard-Stratonovich transformation for the attractive interaction. For example, in some unconventional superconductors such as cuprates, there is no gap structure in the linear absorption spectrum, ${ }^{22}$ unlike in the conventional superconductors studied here. This type of system should be investigated beyond the mean field approximation, as this will be an interesting subject for studying the amplitude mode by our method.

\section{Acknowledgement}

The numerical computation in this work was carried out at the Yukawa Institute Computer Facility. 


\section{References}

1) For example, G. Schön, in Nonequilibrium Superconductivity, ed. D. N. Langenberg and A. I. Larkin (Elsevier, New York, 1986) Chap. 13.

2) P. W. Anderson, Phys. Rev. 112, 1900 (1958).

3) A. J. Leggett, Prog. Theor. Phys. 36, 901 (1966).

4) T. Jujo, J. Phys. Soc. Jpn 81, 044710 (2012).

5) J. Clarke and F. K. Wilhelm, Nature 453, 1031 (2008).

6) A. Schmid, Phys. Kondens. Mater. 8, 129 (1968).

7) A. F. Volkov and S. M. Kogan, Sov. Phys. JETP 38, 1018 (1974).

8) R. Sooryakumar and M. V. Klein, Phys. Rev. Lett. 45, 660 (1980).

9) P. B. Littlewood and C. M. Varma, Phys. Rev. B 26, 4883 (1982).

10) R. Matsunaga, Y. I. Hamada, K. Makise, Y. Uzawa, H. Terai, Z. Wang, and R. Shimano, Phys. Rev. Lett. 111, 057002 (2013).

11) The absorption spectrum of the Mattis-Bardeen ${ }^{17}$ type is observed in the nonlocal limit $\left(v_{F} q \gg \Delta\right)$ or dirty materials $(\alpha \gg \Delta)$. (In the former and latter cases, the absorption occurs because of the nonconservation of the momentum in the propagating direction of external field and the finite damping rate of electrons, respectively.) Here, $1 / q$ is the characteristic length of the external field (the magnetic field penetration depth or thin film thickness). Introducing $q \neq 0$ in calculations to make the absorption finite ${ }^{23,24}$ corresponds to a nonlocal case. The absorption disappears in the clean and local limits $\left(\alpha \ll \Delta\right.$ and $\left.v_{F} q \ll \Delta\right)$.

12) L. V. Keldysh, Sov. Phys. JETP 20, 1018 (1965).

13) A. Kamenev and A. Levchenko, Adv. Phys. 58, 197 (2009).

14) As for the validity of omitting a diamagnetic term $\left(\partial v_{k} / \partial k\right)$, for example, the ratio of $\sum_{k} \int \mathrm{d} \epsilon \operatorname{Tr}\left[G \tau_{3} G \tau_{3}\right]\left(\partial v_{k} / \partial k\right)^{2}$ to $\sum_{k} \int \mathrm{d} \epsilon \operatorname{Tr}[G G G G]\left(v_{k}\right)^{4}$ is proportional to $(1 / m)^{2} /\left(v_{F}^{2} / \Delta\right)^{2}$. Then, replacing $G G v_{k}^{2}$ by $G \partial v_{k} / \partial k$ introduces a small factor $\Delta / E_{F}$ $\left(E_{F}=k_{F}^{2} / 2 m\right)$, and the diamagnetic term is negligible. This is in contrast to the case of Mott insulators $^{25}$ in which the magnitude of the gap is comparable to the band width and the $\partial v_{k} / \partial k$ term is predominant over the $v_{k}$ term.

15) A. A. Abrikosov and L. P. Gor'kov, Sov. Phys. JETP 8, 1090 (1959). 
16) For example, T. Tsuneto, Superconductivity and Superfluidity (Cambridge University Press, New York, 1998) Chap. 4.

17) D. C. Mattis and J. Bardeen, Phys. Rev. 111, 412 (1958).

18) W. Zimmermann, E. H. Brandt, M. Bauer, E. Seider, and L. Genzel, Physica C 183, 99 (1991).

19) G. Baym and L. P. Kadanoff, Phys. Rev. 124, 287 (1961).

20) In the linear response, the quantity corresponding to $\hat{\chi}_{\omega}^{K}$ is written as $\left(2 \pi i N^{3}\right)^{-1} \sum_{\boldsymbol{k}} \int \mathrm{d} \epsilon \hat{\tau}_{3} v_{\boldsymbol{k}}^{x}\left[\hat{G}_{\boldsymbol{k}+\boldsymbol{q} / 2}^{+}(\epsilon+\omega) \hat{G}_{\boldsymbol{k}-\boldsymbol{q} / 2}^{K}(\epsilon)+\hat{G}_{\boldsymbol{k}+\boldsymbol{q} / 2}^{K}(\epsilon+\omega) \hat{G}_{\boldsymbol{k}-\boldsymbol{q} / 2}^{-}(\epsilon)\right] \hat{\tau}_{3}$. Here, the vertex correction by the impurity scattering $\check{I}$ [Eq. (5)] is omitted because this does not affect the conclusion below. The direction of the external field ( $x$ in $v_{\boldsymbol{k}}^{x}$ ) and vector representations for $k$ and $q$ are explicitly written. The vector $\boldsymbol{q}$ of the external potential $A_{\boldsymbol{q}}^{x}(\omega)$ is perpendicular to the $x$-axis for the transverse response, and then the above quantity vanishes. This argument is the same as the discussion about the ineffectiveness of the collective mode for the transverse response in Ref. 26.

21) P. W. Anderson, J. Phys. Chem. Sol. 11, 26 (1959).

22) K. Kamarás, S. L. Herr, C. D. Porter, N. Tache, D. B. Tanner, S. Etemad, T. Venkatesan, E. Chase, A. Inam, X. D. Wu, M. S. Hegde, and B. Dutta, Phys. Rev. Lett. 64, $84(1990)$.

23) T. Papenkort, V. M. Axt, and T. Khun, Phys. Rev. B 76, 224522 (2007).

24) H. Krull, D. Manske, G. S. Uhrig, and A. P. Schnyder, Phys. Rev. B 90, 014515 (2014).

25) T. Jujo, J. Phys. Soc. Jpn 77, 064703 (2008).

26) J. R. Schrieffer, Theory of Superconductivity (Addison-Wesley, Redwood City, Calif., 1983) revised ed., Chap. 8, p. 238. 